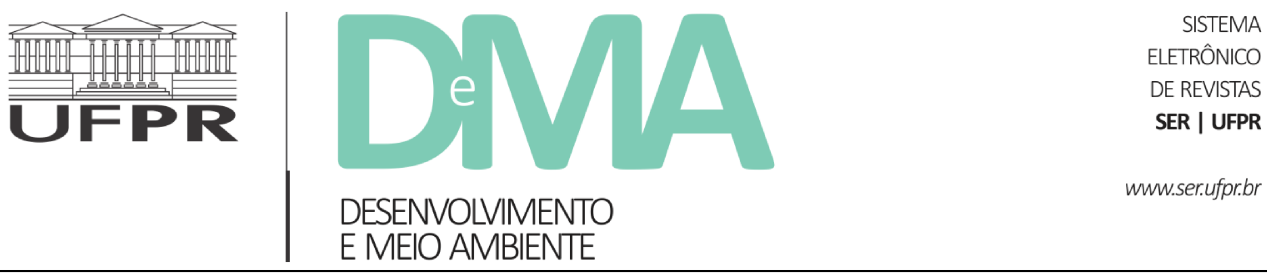

\title{
Análise de programas de pagamento por serviços ambientais no sul do Brasil: identificando estratégias para a conservação da Araucaria angustifolia
}

\section{Analysis of programs of payment for environmental services in southern Brazil: identifying strategies for the conservation of Araucaria angustifolia}

\author{
Mário Muniz TAGLIARI ${ }^{*}$, Victor Augusto MOREIRA², Nivaldo PERONI ${ }^{1}$ \\ ${ }^{1}$ Universidade Federal de Santa Catarina (UFSC), Florianópolis, SC, Brasil. \\ ${ }^{2}$ Fundação CERTI - Centros de Referência em Tecnologias Inovadoras, Florianópolis, SC, Brasil. \\ *E-mail de contato: mario.tagliari@posgrad.ufsc.br
}

Artigo recebido em 13 de julho de 2018, versão final aceita em 7 de março de 2019.

RESUMO:

\begin{abstract}
As estratégias de conservação no Brasil não se limitam à criação de Unidades de Conservação, seja de Proteção Integral ou de Uso Sustentável. Há abordagens distintas, que aliam conservação ao acesso aos serviços ambientais que decorrem da biodiversidade, como por exemplo Pagamento por Serviços Ambientais (PSA). Ao longo da Floresta Ombrófila Mista (FOM), a Araucaria angustifolia, ou Pinheiro-do-Paraná, encontra-se tão ameaçada quanto o próprio ecossistema no qual está inserida. Com o intuito de avaliar os programas de PSA criados na FOM que contribuem na conservação da espécie, este estudo avaliou quatro programas de PSA: "Desmatamento Evitado", "Araucária+", "Corredor Ecológico Chapecó" e "Estrada com Araucárias", distribuídos nos estados do Paraná e Santa Catarina. Foi feita uma revisão bibliográfica para a seleção desses programas e, uma vez selecionados, foram levantados seus indicadores e tipologias, além de ser feito um índice apontando pontos positivos e negativos entre os programas selecionados. Esses projetos possuem objetivos amplos, que valorizam os indicadores de adicionalidade, permanência, transação, e com pouca evidência ao indicador de vazamento (spillover). As tipologias são contempladas em sua maioria, como suporte e regulação, porém os serviços culturais poderiam ser contemplados com uma frequência maior, por exemplo. Por fim, ao analisar esses quatro projetos, foi observado que, apesar de poucos indicadores e tipologias que envolvem um PSA, há possibilidades distintas para aliar conservação e valoração da biodiversidade positivamente, pois os programas possuem metodologias e objetivos distintos em geral.
\end{abstract}


Palavras-chave: PSA; Araucária; conservação; indicadores; tipologias.

ABSTRACT: Brazilian's conservation strategies are not limited only by the creation of Protected Areas, either Integral Protection or Sustainable Use. There are distinct strategies that aim to value services provided by ecosystems, combining conservation to the access of environmental services provided by biodiversity. In this context, there are the Payments for Environmental Services (PES). Throughout the Mixed Ombrophilous Forest (MOF), the plant species Araucaria angustifolia, known as Pinheiro-do-Paraná, is as threatened as the MOF ecosystem itself. Consequently, PES programs appear as an alternative tool to the main strategies for the preservation and conservation of biodiversity. In order to evaluate the PES programs created at the MOF that contribute to the species' conservation, we evaluated four PES projects in southern Brazil: "Desmatamento Evitado", "Araucária+", "Corredor Ecológico Chapecó" and "Estrada com Araucárias", all of them distributed in Paraná and Santa Catarina states. The basis for the programs' selection was a literature review. Once we selected them, we identified the indicators and topologies of each one, as well as an index indicating positive and negative points between the selected PES programs. The results showed that the selected PES have broad objectives, which value differently the indicators of additionality, permanence, transaction, but showed little evidence to the spillover indicator. The mostly contemplated typologies are support and regulation. Cultural services typology could be contemplated more frequently, for instance. Finally, by analyzing these four programs, despite few indicators and typologies that involve a PES, there are different possibilities that align conservation and valuation of biodiversity positively, since the programs have different methodologies, scopes, and objectives in general.

Keywords: PES; Araucaria; conservation; indicators; typologies.

\section{Introdução}

A Mata Atlântica é o segundo maior bioma de floresta tropical da América do Sul, cuja cobertura já atingiu aproximadamente 1,5 milhões de $\mathrm{Km}^{2}$ ao longo da costa brasileira, estendendo-se para regiões que abrangem áreas na Argentina e no Paraguai (Ribeiro et al., 2009). Acredita-se que sua biodiversidade de mais de 20.000 espécies vegetais seja maior do que da própria floresta amazônica (Colombo \& Joly, 2010). Aproximadamente 50\% das espécies são endêmicas (Myers et al., 2000).

Um grande desafio para a conservação desse bioma deve-se ao fato de que $60 \%$ da população brasileira vive na sua faixa de distribuição, produ- zindo $70 \%$ do produto interno bruto nacional e $66 \%$ da economia industrial do país, além de contar com suas duas maiores capitais: São Paulo e Rio de Janeiro (Scarano \& Ceotto, 2015). Por consequência, a degradação dos ecossistemas naturais é a principal causa de perda de biodiversidade, ao combinar urbanização, industrialização e expansão agrícola dentro do domínio da Mata Atlântica (Scarano \& Ceotto, 2015). Tal cenário de degradação tem como consequência um alto índice de espécies ameaçadas e/ou em risco de extinção (Ribeiro et al., 2009; 2011), classificando-a como um hotspot. Um fator adicional mais recente, os efeitos das mudanças climáticas (Colombo \& Joly, 2010; Lemes \& Loyola, 2013; Ferro et al., 2014; Scarano \& Ceotto, 2015), contribuiu para classificá-la entre os 3 hotspots mais 
vulneráveis às mudanças climáticas (Bellard et al., 2014), dentre 35 no mundo, mesmo com mais de 700 Unidades de Conservação (UC) ao longo de sua distribuição (Galindo-Leal \& Câmara, 2003), mas que ocupam apenas $1,5 \%$ de sua área total (Ribeiro et al., 2009).

Há três fitofisionomias predominantes na Mata Atlântica: Floresta Ombrófila Densa (FED), Florestas Estacionárias Decíduas e Semidecíduas (FEDS) e a Floresta Ombrófila Mista (FOM), também conhecida como Floresta ou Mata de Araucárias. A FOM inicialmente possuía 200.000 $\mathrm{Km}^{2}$, distribuindo-se principalmente nos estados do Paraná - PR (40\% do território), Santa Catarina - SC (30\%) e Rio Grande do Sul - RS (25\%), além disso, segundo Carvalho (1994), a Floresta de Araucárias apresentava pequenas manchas em regiões específicas nos estados do Rio de Janeiro, São Paulo e Minas Gerais (ver também Reis et al., 2014). As espécies distribuídas na FOM estão adaptadas a temperaturas mais amenas, com geadas frequentes no inverno (Roderjan et al., 2002; Wrege et al., 2016), encontrando-se numa ampla faixa de altitude, de 500 a 1200 metros (Duarte et al., 2012). A espécie mais emblemática é a Araucaria angustifolia, popularmente conhecida como Araucária, Pinheiro ou Pinheiro-do-Paraná, sendo a espécie dominante do dossel da FOM, devido a sua altura elevada e formato da copa em candelabro (Gasper et al., 2013; Meyer et al., 2013; Adan et al., 2016).

A exploração madeireira intensiva da Araucária ao longo do século $\mathrm{XX}$, que apesar de ter sido um aspecto economicamente relevante no Sul do Brasil, somada à expansão agrícola e, posteriormente, à urbanização, causou a drástica redução das populações da espécie (Da Silva \& Reis, 2009) e dos remanescentes originais da FOM, restando de 5\%
(Guerra et al., 2002) até 12\% (Ribeiro et al., 2009). Analisando imagens geradas por satélites e dados de campo, Vibrans et al. (2013) estimou com maior precisão a área remanescente da FOM, concluindo que menos de $25 \%$ da distribuição original persiste. Esse cenário de exploração, expansão agrícola e urbanização permite classificar essa fitofisionomia como muito ameaçada (Kanieski et al., 2010). A superexploração permitiu que mecanismos legais fossem utilizados para a conservação da espécie, devido a sua relevância ecológica-cultural (Machado Mello \& Peroni, 2015), como a Resolução CONAMA n 278 (Conama, 2001), que proíbe o corte e exploração de espécies ameaçadas de extinção, flexibilizando o uso sustentável de exploração uma vez estabelecidos critérios técnicos e científicos (Da Silva \& Reis, 2009), assim como a Lei No 11.428 de 2006, que dispõe sobre a utilização e conservação da vegetação da Mata Atlântica (Brasil, 2006).

$\mathrm{O}$ uso, manejo e consumo das sementes (pinhão) da Araucária historicamente tem moldado paisagens culturais e transformando o ecossistema da FOM, com forte influência de comunidades locais distribuídas no Sul do Brasil (Reis et al., 2014; Machado Mello \& Peroni, 2015; Adan et al., 2016; Zechini et al., 2018; Lauterjung et al., 2018). O consumo do pinhão como recurso alimentício, a exploração histórica da espécie, além da influência cultural e ambiental na área da FOM, são exemplos de como o uso, manejo e conservação da Araucária fornecem, direta ou indiretamente, serviços ambientais. O cenário de instabilidade, degradação e fragmentação ambiental da FOM (Vibrans et al., 2011) pode influenciar, contudo, na qualidade e funcionalidade dos serviços ambientais fornecidos por esse importante ecossistema. 


\subsection{Serviços ambientais na Floresta Ombrófila Mista}

Serviços ambientais (ou também serviços ecossistêmicos) são definidos por Daily (1997, p. 3) como "condições e processos, através dos quais os ecossistemas, e as espécies que fazem parte dele, sustentam a vida humana no planeta". Os serviços ambientais geram benefícios resultantes do funcionamento saudável dos ecossistemas, que são apropriados direta ou indiretamente para e pelos seres humanos (Costanza, 1998; De Groot et al., 2002), no presente ou no futuro (Daly \& Farley, 2004). Apesar do emprego de conceitos distintos para serviços ambientais e serviços ecossistêmicos, os argumentos utilizados para defini-los se alternam de acordo com quem os define (Chaudhary et al., 2015). Isso se deve à constante evolução do conceito, que em um primeiro momento configurava-se em um aspecto mais acadêmico, sobretudo entre ecólogos e economistas, porém, no início do século XX, foi sendo incorporado às agendas políticas e sociais de modo constante, tornando seu significado multidisciplinar, institucional e global (Chaudhary et al., 2015). Desse modo, serviços ambientais e serviços ecossistêmicos podem ser conceituados de modo similar (Prado, 2014; Bernardo, 2016).

Os serviços ambientais são agrupados em quatro classificações distintas, segundo a Avaliação Ecossistêmica do Milênio (AEM): (i) de regulação - relacionado com a qualidade e manutenção dos processos ecossistêmicos; (ii) de provisão - que é o produto dos ecossistemas, como alimentos, recursos madeireiros, fibras etc.; (iii) culturais - que contribuem com valores étnicos e sociais de comunidades; e (iv) de suporte - que é a contribuição para outros serviços ecossistêmicos, seja ciclagem de nutrientes, qualidade do solo, dispersão de sementes (AEM, 2005).

A FOM fornece diversos serviços ambientais, dentre eles os de suporte e regulação, que possuem importante papel na manutenção dos ecossistemas, do clima e dos ciclos biogeoquímicos (Joly et al., 2014). Quanto aos serviços de provisão, pode-se destacar na provisão de alimentos e recursos fitogenéticos (Vieira da Silva \& Reis, 2009). A Araucária e a erva-mate (Ilex paraguariensis), plantas nativas da FOM, por exemplo, produzem recursos alimentícios para sua fauna associada e para a sociedade, além de fornecerem serviços culturais importantes, visualizados por meio do hábito do consumo do pinhão e de beber chimarrão (Souza et al., 2010; Moreira, 2014).

\subsection{Pagamento por Serviços Ambientais}

A utilização de mecanismos econômicos integrados à valoração dos serviços ambientais tornou-se prática recorrente como estratégia de conservação e como alternativa complementar à conservação biológica, uma vez que geram ativos econômicos pelos serviços ambientais fornecidos (Daily et al., 2000).

Um dos mecanismos econômicos são os Pagamentos por Serviços Ambientais (PSA). Os PSA são definidos por Wunder $(2005$, p. 3) como "uma transação voluntária ou um pagamento que um ou mais beneficiários de serviços ambientais fornecem para um provedor destes serviços ambientais, caso este provedor assegurar a provisão destes serviços por um longo período de tempo". Dessa forma, um fornecedor de um serviço 
ambiental (como um agricultor típico da FOM) poderia mudar seu comportamento se o custo adicional da mudança (conservação da biodiversidade) fosse coberto pelo incentivo econômico ao serviço prestado (Wunder, 2005).

No escopo de um projeto de PSA, é preciso, necessariamente, ser identificado dentro do seu arranjo e governança: (i) ao menos um serviço ambiental bem definido; (ii) ao menos um fornecedor; (iii) ao menos um comprador; (iv) transações voluntárias; e (v) evidência de condicionalidade (Wunder, 2005). Além disso, indicadores de qualidade são encontrados nos PSA, influenciando na efetividade dos programas (Santos \& Silvano, 2016). Podem ser encontrados como indicadores de efetividade nos PSA: (i) adicionalidade - o programa de PSA deve induzir mudanças no uso da terra e da água, em propriedades rurais; (ii) custo de transação - levantamento de todos os valores decorrentes do processo de criação do PSA: negociação, monitoramento, controle, ou seja, que não são os pagamentos propriamente ditos; (iii) custo de oportunidade - é o cálculo do ônus (ou bônus) em troca da produção de um produto pelo fornecimento de outro, nesse caso, o PSA; (iv) permanência - que é a continuidade do serviço ambiental após o encerramento dos pagamentos; (v) vazamento - que é o deslocamento de um gerador de externalidades negativas para uma área além da alcançada pelo PSA (Wunder, 2005; Young et al., 2007; Engel et al., 2008; Wunder et al., 2009; White \& Minang, 2011; Plumb et al., 2012; Wunder, 2015; Santos \& Silvano, 2016).

Para a conservação da Araucária, os PSA podem ser uma opção alternativa à estratégia comumente utilizada para conservação no Brasil, que são as Unidades de Conservação (UC). Na FOM, a influência humana no manejo da Araucária, seja histórica (Bitencourt \& Krauspenhar, 2006) ou atual (Adan et al., 2016) - principalmente por pequenos agricultores, moldou historicamente sua paisagem (Reis et al., 2014). A demarcação de UCs nessa fitofisionomia também causou e ainda gera conflito de interesses entre conservação da biodiversidade e uso e manejo de recursos por agricultores. No entanto, a influência humana pode contribuir para gerar serviços ambientais diretos e indiretos (Brandt, 2012; Peroni et al., 2013; Machado Mello \& Peroni, 2015; Adan et al., 2016), principalmente pelas ações que favorecem continuamente o interesse pelo pinhão, por meio de seu consumo, ocorrendo em áreas de entorno de UCs, como na região serrana do estado de Santa Catarina por meio de sistemas tradicionais de manejo (Adan et al., 2016; Zechini et al., 2018). Os PSA para essa região são apontados como um mecanismo que permite um maior equilíbrio de interesses do que apenas dentro do limitado espaço delimitado por áreas protegidas (i.e. UC) que restrinjam o uso sustentável da espécie e seus recursos. Com base nessa abordagem teórica dos PSA e da valoração econômica da biodiversidade, há projetos e propostas de PSA, tanto públicos quanto privados, que são desenvolvidos com o intuito de conservação da biodiversidade na FOM. Há bastante diversificação de PSA, tanto que projetos e/ou iniciativas de PSA na FOM, focando especificamente e/ou envolvendo a Araucária, já foram criados, estão estabelecidos e/ou serão desenvolvidos. É com base nesses projetos já criados, focando nos que estão por vir, que este trabalho pretende contribuir. Dessa forma, quais são as características dos programas de PSA na Floresta Ombrófila Mista quanto à conservação da 
Araucaria angustifolia? Quais atributos e indicadores se destacam do ponto de vista de efetividade desses programas? Como otimizar novos projetos de PSA? Analisando estudos de caso de PSA implementados atualmente, pretendemos identificar as principais estratégias de valoração econômica dos PSA em andamento na FOM, assim como seus principais pontos positivos e negativos e propor sugestões para projetos futuros de Programas de Pagamento por Serviços Ambientais.

\section{Material e métodos}

\subsection{Seleção dos projetos de PSA}

Para a fundamentação teórica e seleção dos projetos, foi realizado, inicialmente, levantamento por palavras-chave (Araucária, FOM, Pagamentos por Serviços Ambientais) via plataformas de base de dados de produção científica: Portal de Periódicos Capes, Web of Science e os Repositórios Digitais Institucionais da Universidade Federal do Paraná, Universidade Federal de Santa Catarina e Universidade Tecnológica Federal do Paraná. Como base complementar às plataformas, foi usado também o Google Scholar.

O critério de seleção de projetos baseou-se no cumprimento de algumas premissas: (i) remuneração dos envolvidos já estabelecida e com metodologia definida (original ou não); (ii) arranjo institucional firmado, ou seja, acordo de parceria público-privada, apenas privada ou apenas pública; e (iii) projetos necessariamente envolvendo o pagamento por serviços ambientais que envolvam a conservação e/ ou ao uso de recursos florestais não madeireiros da Araucaria angustifolia de modo sustentável. Além disso, a disponibilidade de informações referentes à abrangência do PSA, o tipo de serviço ambiental provido, o arranjo institucional, o valor potencial dos pagamentos/remuneração e o público-alvo foram pré-requisitos para a escolha definitiva dos programas. Essas informações foram complementadas também pelo acesso a documentos públicos e, obrigatoriamente, com acesso gratuito (Fundação do Meio Ambiente - FATMA, Fundação Grupo O Boticário, Centros de Referência em Tecnologias Inovadoras [CERTI], Sociedade de Pesquisa em Vida Selvagem e Educação Ambiental - SPVS, Secretaria do Meio Ambiente e Recursos Hídricos - SEMA). Essa etapa permitiu elaborar um quadro comparativo entre os programas segundo metodologia aplicada por Santos \& Silvano (2016).

De acordo com os métodos acima descritos, foram selecionados os quatro projetos de PSA: 1) O "Programa Desmatamento Evitado", desenvolvido pela Sociedade de Pesquisa em Vida Selvagem e Educação Ambiental - SPVS, por meio da criação de Reservas Particulares do Patrimônio Natural - RPPN (Seehusen et al., 2011); 2) A implantação de um PSA no "Corredor Ecológico Chapecó - Santa Catarina"; desenvolvido no âmbito do Programa de Recuperação Ambiental e Apoio ao Pequeno Produtor Rural (Projeto Microbacias 2, sob responsabilidade da FATMA - Fundação do Meio Ambiente; Alarcon et al., 2013); 3) A "Iniciativa Araucária+", no Planalto Serrano Catarinense, entre os municípios de Painel, Lages, Urubici, Urupema e Bom Jardim da Serra com a Bonificação por Produção Sustentável, executados em parceria pela Fundação Grupo Boticário de Proteção à Natureza e Fundação Certi (Moreira, 2014); 4) Projeto Sistemas Estaduais de PSA no Paraná e Santa Catarina: análise do 
"Projeto Estradas com Araucárias", parte do Programa Bioclima da Secretaria do Meio Ambiente - SEMA e Instituto Ambiental do Paraná - IAP (Santos \& Silvano, 2016).

\subsection{Avaliação da efetividade dos PSA}

Para avaliar a efetividade de um PSA, uma série de fatores foram considerados. Primeiramente, a existência de adicionalidade, ou seja, se a atividade do projeto de PSA gera mudanças no uso da terra (ou água) por parte dos proprietários rurais (Engel et al., 2008). Outro conceito avaliado foi o de permanência, que é o potencial de continuidade de manutenção da qualidade do serviço ambiental prestado durante e após o fim do programa de PSA. Também foram identificados os custos de transação, ou seja, são todos os custos que não são os pagamentos propriamente ditos, como: monitoramento, controle, fiscalização e validação dos serviços ambientais definidos (Wunder et al., 2009; Santos \& Silvano, 2016). Foram avaliados a existência da abordagem de compensação do custo de oportunidade: analisar se os programas levam em conta o custo de oportunidade do uso da terra nos processos de valoração dos serviços ambientais e respectivos pagamentos (Young et al., 2007; White \& Minang, 2011; Plumb et al., 2012) e, por fim, o indicador de vazamento, que é identificado quando atividades geradoras de externalidades negativas são deslocadas para além da abrangência de um programa de PSA implementado (Wunder, 2005).

Para cada projeto avaliado, foi atribuída uma nota para a presença do indicador (nota 1) e para sua ausência (nota 0 , zero). No caso de não haver informações suficientes, foi considerado como "não avaliado" (NA). Do mesmo modo, as classificações de tipologia de serviços (regulação, provisão, cultural e suporte) foram usadas para analisar os projetos de PSA selecionados, sendo utilizada uma adaptação da abordagem apresentada por Santos \& Silvano (2016), que utilizou apenas os indicadores (adicionalidade, custo de transação, custo de oportunidade e vazamento).

\section{Resultados e discussão}

\subsection{O "Programa Desmatamento Evitado"}

Quanto ao "Programa Desmatamento Evitado" (Tabela 1), criado pela SPVS, em 2003, um dos objetivos principais é a conservação da Mata de Araucárias, além da proteção de remanescentes ambientais no sul do país e de restaurar áreas degradadas (Seehusen et al., 2011; SPVS, 2013), com projetos nos municípios de Santa Catarina (Itaiópolis e Alfredo Wagner) e do Paraná (Ponta Grossa, Lapa e Prudentópolis).

É um programa que valoriza a continuidade de seus projetos, buscando meios para estenderem, a longo prazo, determinados PSA, servindo de base como modelo para ser seguido pelo Poder Público (SPVS, 2013).

\subsection{O PSA no "Corredor Ecológico Chapecó"}

Implementado em 2014 pela Fundação do Meio Ambiente de Santa Catarina/Instituto do Meio Ambiente de Santa Catarina (FATMA/IMA), EPAGRI - Empresa de Pesquisa Agropecuária e 
TABELA 1 - Descrição resumida do PSA do Programa Desmatamento Evitado ${ }^{1}$

\begin{tabular}{ll}
\hline Abrangência & Paraná e Santa Catarina (principalmente) e até 5000 hectares (ha) conservados ${ }^{1}$. \\
\hline Serviço Ambiental em destaque & $\begin{array}{l}\text { Estocagem de carbono; } \\
\text { Conservação fauna e flora; } \\
\text { Criação de RPPN }{ }^{2} .\end{array}$ \\
\hline Arranjo Institucional & $\begin{array}{l}\text { Execução e monitoramento: SPVS; } \\
\text { Financiadoras: setor privado. } \\
\text { Convênios: modelo público-privado } 2 .\end{array}$ \\
\hline $\begin{array}{l}\text { Valor potencial do PSA e metodologia de } \\
\text { valoração ambiental }\end{array}$ & R $\$ 500,00$ ha/ano. Envolvendo valor de repasse ao proprietário e a gestão do projeto1. \\
\hline Tipos de Remuneração & Empresas privadas "adotam" a área preservada e proprietários recebem "premiação". \\
\hline Público-alvo & Pequenos Agricultores - áreas privadas. \\
\hline
\end{tabular}

${ }^{1}$ FONTE: Seehusen et al. (2011); ${ }^{2}$ SPVS (2013).

Extensão Rural de Santa Catarina e a Secretaria do Estado do Desenvolvimento Econômico Sustentável (SDS), dentro do projeto Programa Santa Catarina Rural, esse PSA (Tabela 2) destina recursos financeiros a proprietários rurais que conservam áreas de mata considerada nativa ou que pretendem recuperar áreas degradadas. Meta de alcançar 1000 ha de áreas preservadas (Alarcon et al., 2013).

\subsection{Programa "Araucária+: Bonificação por Produção Sustentável”}

A iniciativa "Araucária+" visa à conservação da biodiversidade da FOM sob o enfoque do Padrão Sustentável de Produção de Erva-mate e Pinhão (Fundação Certi, 2014), em que, juntamente com esse modelo, o método de Bonificação por Produção Sustentável (Tabela 3) é a ferramenta de

TABELA 2 - Descrição simplificada do PSA do CE Chapecó. Adaptado da secretaria de estado da agricultura e da pesca².

\begin{tabular}{ll}
\hline Abrangência & Santa Catarina (regiões oeste e planalto norte); extensão futura de até 1000 ha. \\
\hline Serviço Ambiental em destaque & $\begin{array}{l}\text { Conservação de mata nativa e recuperação de áreas degradadas da FOM em exceden- } \\
\text { tes de áreas de preservação e reserva legal. }\end{array}$ \\
\hline Arranjo Institucional & $\begin{array}{l}\text { Execução e monitoramento: FATMA; } \\
\text { Financiadoras: setor público (sds), com recursos do Banco Mundial; } \\
\text { Projetos renováveis por até } 3 \text { anos. }\end{array}$ \\
\hline Valor potencial do PSA e metodologia de & $\begin{array}{l}\text { De R } 87,50 \text { até R } \$ 350 \text { por ha. } \\
\text { Utiliza a Método Oásis }{ }^{1} .\end{array}$ \\
valoração ambiental & Compensação por hectare preservado ou recuperado. \\
\hline Tipos de Remuneração & Pequenos agricultores - áreas privadas e participação voluntária \\
\hline Público-alvo &
\end{tabular}

\footnotetext{
${ }^{2}$ SCRural (2014).
} 
PSA criada para fomentar economicamente a não extração do pinhão e erva-mate, além da restrição à prática da pecuária extensiva, em remanescentes da Mata de Araucárias com alta relevância para a conservação. (Fundação Certi, 2014).

\subsection{Projeto "Estradas com Araucárias e o Programa Bioclima"}

Esse projeto de PSA(Tabela 4) é um incentivo ao plantio de araucárias em divisas de propriedades rurais familiares com faixas de domínio de estradas (Oliveira, 2015). É um projeto de compensação, no qual empresas privadas pagam pelas emissões de gases de efeito estufa (GEE) a produtores rurais que plantam araucárias ao longo de suas divisas com estradas. Isso permite uma série de benefícios indiretos, como conservação, conectividade entre áreas protegidas, paisagismo, produção de pinhão, recursos para fauna silvestre entre outros (Oliveira, 2015). O projeto surgiu em 2011 e contempla mais de 60 unidades nos municípios do Paraná (Lapa e Irati) e Santa Catarina (Caçador).

Ao avaliar os serviços ambientais contemplados pelos programas selecionados, nota-se que o potencial ecológico da Araucária é explorado por diferentes maneiras (Tabela 6), seja por meio dos serviços de regulação: estratificação do dossel da FOM (Ribeiro et al., 2013) ou regeneração florestal em áreas de clareiras, devido ao seu caráter heliófilo (Avila et al., 2016). Também, pelos serviços

TABELA 3 - Descrição simplificada do PSA da iniciativa “Araucária+”, por meio do método de Bonificação por Produção Sustentável³.

$\begin{array}{ll}\text { Abrangência } & \text { Santa Catarina (região serrana): } \\ & \text { Urubici, Lages, Urupema, Painel e Bom Jardim da Serra. }\end{array}$

Serviço Ambiental em destaque

Manutenção de remanescentes da FOM não utilizados para extração do pinhão e/ou pecuária.

\section{Arranjo Institucional}

Valor potencial do PSA e metodologia de valoração ambiental
A Gestão do programa é realizada pela Fundação CERTI, e o recurso para os pagamentos deriva das empresas integradas à iniciativa Araucária+, em que destinam um percentual de recursos para o programa de PSA em cada transação comercial realizada dentro do Padrão Sustentável de Produção.
Tipos de Remuneração

Público-alvo
De $\mathrm{R} \$ 69,13$ até $\mathrm{R} \$ 414,79$ por ha/ano, com potencial ao longo do tempo de ampliar para $\mathrm{R} \$ 829,58$.

Inspirado no Método Oásis

${ }^{3}$ Fundação Certi (2014); Moreira (2014). 
ambientais de provisão: como o uso histórico da madeira ou pelo manejo e comércio do pinhão. $\mathrm{O}$ manejo e comércio do pinhão, tanto atual quanto historicamente, além da paisagem da FOM por si só, refletem em uma identidade cultural local, principalmente no sul do Brasil, onde a Araucária e o pinhão são símbolos de municípios, estados ou mesmo de festas culturais tradicionais (Peroni et al., 2013; Reis et al., 2014; Machado Mello \& Peroni, 2015). Assim, ações e/ou programas que visem à conservação da Araucária favorecem a existência e permanência de serviços culturais, como o envolvimento de pequenos agricultores no uso, manejo e comércio de pinhões pelo sul do Brasil, atividade que denota um estilo de vida que valoriza as tradições e o meio ambiente do sul do país (Reis et al., 2014). Além disso, a dispersão de sementes da Araucária pode ser classificada como serviço ambiental de suporte (ver Iob \& Vieira, 2008;
Wrege et al., 2016), além de servir como alimento para a fauna (Duarte et al., 2002; Bogoni et al., 2018). A prestação desses serviços ambientais, direta ou indiretamente, é um aspecto positivo dos PSA apresentados, pois fornece garantia e suporte à espécie, de modo que os serviços ambientais continuem a serem fornecidos nos locais onde se distribuem.

Wunder (2005) destaca que para verificar se os programas de PSA estão atingindo seus objetivos, eles devem satisfazer o máximo de indicadores de efetividade (ver Tabela 5). Com base na literatura, verificou-se que os programas selecionados devem apresentar, no mínimo, adicionalidade, ou seja, devem resultar em ações que induzam mudanças no uso da terra ou água por parte dos proprietários rurais em prol da conservação de um ativo ambiental (Engel et al., 2008), como a Araucária na FOM. A adicionalidade,

TABELA 4 - Descrição simplificada do PSA do "Projeto Estradas com Araucárias"4.

Abrangência Municípios do Paraná e Santa Catarina

Serviço Ambiental em destaque

Arranjo Institucional

Valor potencial do PSA e metodologia de valoração ambiental
Preservação da araucária, corredores verdes, produção de pinhão, estocagem de carbono, beleza cênica.
Tipos de Remuneração

Público-alvo

Propriedades agrícolas com faixas em domínios de estradas no PR e SC.

Compensação financeira pela estocagem de GEE via pagamento direto.

${ }^{4}$ Oliveira (2015).

Até 200 mudas plantadas por propriedade com bonificação de R\$5,00/muda. Máximo de $\mathrm{R} \$ 1.000,00 /$ propriedade, até fase produtiva da araucária. 
entretanto, não deve ser premissa obrigatória, pois podem haver agricultores que conservam os remanescentes florestais em suas propriedades sem os benefícios financeiros de um programa de PSA. Agricultores na FOM, por exemplo, que se encaixem nesse caso, prestam serviços ambientais sem compensação de um PSA, e poderiam ser contemplados porque, ao contrário, um PSA apenas compensaria aqueles que anteriormente geraram danos para o ecossistema (Seehusen et al., 2011; Santos \& Silvano, 2016). Esse caso específico deve ser valorizado, no qual um programa de PSA premiaria produtores/agricultores que já realizam ações de conservação e/ou restauração não somente por obrigação legal, mas também por motivações próprias.
$\mathrm{O}$ indicador de monitoramento de vazamento não foi descrito e/ou identificado nos programas selecionados. Como esse indicador requer monitoramento direto fora do alcance geográfico do programa criado, ou seja, além da abrangência real do PSA criado, esse atributo deve ser adicionado a novos programas com menor frequência. Entretanto é possível que em um contexto de recursos financeiros reduzidos, o monitoramento além da área de abrangência de um PSA possa encarecer ainda mais a sua manutenção, tendo, por consequência, influência no indicador de permanência - que é a continuidade da prestação do serviço ambiental após o fim do programa. Entretanto são poucos os trabalhos que discutem essa situação, tampouco sistematizam criticamente os instrumentos de

TABELA 5 - Indicadores avaliados $(0$ = ausência de indicador, $1=$ presença de indicador, NA = não-avaliado, por não encontrar informações) para os PSA na FOM envolvendo a Araucária. As tipologias foram classificadas após a interpretação da descrição das atividades e resultados de cada programa ${ }^{5}$.

\begin{tabular}{|c|c|c|c|c|c|c|}
\hline PSA na FOM & $\begin{array}{l}\text { Adicionali- } \\
\text { dade }\end{array}$ & $\begin{array}{l}\text { Oportuni- } \\
\text { dade }\end{array}$ & Transação & $\begin{array}{l}\text { Perma- } \\
\text { nência }\end{array}$ & $\begin{array}{l}\text { Vaza- } \\
\text { Mento }\end{array}$ & $\begin{array}{l}\text { Tipo- } \\
\text { logia }\end{array}$ \\
\hline $\begin{array}{l}\text { Programa Desmatamento } \\
\text { Evitado }\end{array}$ & 1 & 1 & 1 & 1 & 0 & $\begin{array}{l}\text { Provisão, regu- } \\
\text { lação, suporte e } \\
\text { cultural }(+4)\end{array}$ \\
\hline
\end{tabular}

$\begin{array}{llllll}\begin{array}{l}\text { PSA Corredor Ecológico } \\ \text { de Chapecó }\end{array} & 1 & 1 & 1 & 1 & 0\end{array}$

\begin{tabular}{|c|c|c|c|c|c|c|}
\hline Programa Araucária+ & 1 & 1 & 1 & 1 & 0 & $\begin{array}{l}\text { Provisão, regu- } \\
\text { lação, suporte e } \\
\text { cultural }(+4)\end{array}$ \\
\hline $\begin{array}{l}\text { Projeto Estradas com } \\
\text { Araucária }\end{array}$ & 1 & 0 & 1 & 1 & NA & $\begin{array}{l}\text { Provisão e su- } \\
\text { porte }(+2)\end{array}$ \\
\hline
\end{tabular}

\footnotetext{
${ }^{5}$ Santos \& Silvano (2016).
} 
PSA no Brasil (Seehusen et al., 2011). Ainda, em relação ao indicador de permanência, de acordo com os documentos disponíveis dos programas de PSA analisados, apenas o programa "Desmatamento Evitado" destacou o objetivo de estender seu programa. Programas de curto prazo podem ter sua eficácia limitada, inclusive se não houver programação de atividades após o término de vigência do PSA (Pagiola et al., 2007), além de que a suspensão temporária de pagamentos pode levar a um desestímulo à conservação por parte dos beneficiários (Santos \& Silvano, 2016). O alcance físico do programa "Desmatamento Evitado" também foi o maior dentre os PSA escolhidos, com até 5.000 ha para uso potencial como PSA, além de objetivar a criação de Reservas Particulares do Patrimônio Natural - RPPN nos locais com o PSA já estabelecido.

Os custos de transação, que são os custos extras além do pagamento pelo serviço propriamente dito, como fiscalização, viabilidade prévia, validação dos serviços providos durante a existência do programa e negociação entre envolvidos (Wunder et al., 2009), foram identificados em todos os programas. No caso do PSA "Corredor Ecológico de Chapecó", foram identificadas propriedades que possuem áreas naturais excedentes às áreas de preservação permanente (APP) e Reserva Legal (RL), em zonas dentro de potenciais ou já existentes corredores ecológicos (SCRural, 2014), envolvendo indiretamente, análises de viabilidade prévia para potenciais locais que recebam o pagamento.

Dentre os indicadores avaliados, é o custo de oportunidade que deve atrair a atenção dos proprietários, pois no mesmo local onde há o serviço ambiental relevante, também existe um local de uso alternativo de recursos, como água e solo (Santos \& Silvano, 2016). Do mesmo modo, todos os programas apresentam custos de oportunidade relativos, porém de modos distintos. O PSA "Estradas com Araucária", por exemplo, oferece, ao máximo, $\mathrm{R} \$ 1000,00$ ha/ano/propriedade.

Dois programas utilizam a metodologia Oásis (Young \& De Bakker, 2014) para o cálculo do valor de pagamento do PSA: "Araucária+" e o Projeto "Corredor Ecológico Chapecó”. Essa metodologia apresenta a função de valoração adaptável a distintos cenários e contextos, levando em consideração o custo de oportunidade destinado pela conservação, premiando os serviços ambientais identificados na propriedade e/ ou adoção das melhores práticas na agricultura (Young \& De Bakker, 2014).Nesses programas, o custo de oportunidade é valorado por meio de uma porcentagem. Esse valor é somado ao cálculo de pagamento, oscilando entre $\mathrm{R} \$ 69,13$ até $\mathrm{R} \$ 414,79$ ha/ano, utilizando $25 \%$ do custo de oportunidade, como por exemplo no projeto PSA "Araucaria+" - Bonificação por Produção Sustentável (Moreira, 2014). A vantagem de ter uma metodologia específica e, nesse caso, com valoração do custo de oportunidade, possibilita aumentar a porcentagem do custo de oportunidade de acordo com o PSA (Moreira, 2014).

As tipologias dos serviços ambientais encontrados em cada programa de PSA: provisão, suporte, regulação e cultural (AEM, 2005) foram identificadas para cada projeto analisado. Quanto aos serviços de provisão e de regulação, com exceção do programa "Estradas com Araucárias", fosse direta ou indiretamente, todos apresentaram serviços que podem produzir/reciclar os elementos estruturais dos ecossistemas, como fontes de ener- 
gia, recursos genéticos e medicinais (i.e. provisão) e promover a continuidade e desenvolvimento dos processos ecológicos (i.e. regulação; Rebollar et al., 2013). Os serviços de suporte, ou seja, ciclagem da água, fotossíntese, formação do solo, ciclagem de nutrientes e o desenvolvimento dos ecossistemas, puderam ser identificados em todos os programas. Finalmente, o serviço cultural, que está relacionado com os benefícios imateriais para o bem-estar humano, além da beleza cênica dos ecossistemas (AEM, 2005), não foi explicitamente discutido no programa Estrada com Araucárias.

\section{Conclusão e recomendações finais}

A partir da análise dos programas de PSA, verificou-se que os serviços providos na Floresta Ombrófila Mista envolvendo a Araucária vêm sendo utilizados dentro do escopo de valoração e monitoramento dos arranjos de PSA. O Projeto "Desmatamento Evitado", por exemplo, usa o PSA para fomentar a criação de RPPNs, que podem aumentar a permanência de um programa, fornecendo a longo prazo serviços de provisão, suporte, regulação e mesmo culturais. Seria muito benéfico se mais PSA tivessem como objetivo final a criação de novas RPPNs. Entretanto, a questão da permanência do PSA quando conciliado com a criação de RPPN, pode ser negativa do ponto de vista econômico para os proprietários, uma vez que o proprietário abdica do uso financeiro de sua propriedade uma vez transformada em RPPN, diminuindo muitas vezes o valor de mercado devido à restrição característica desses refúgios.

Os PSA não devem ter como foco apenas a recuperação de áreas degradadas, pois há uma parcela de pequenos agricultores que conservam seus remanescentes por meio do uso e manejo sustentável dos recursos. Nesse caso, os PSA podem servir como fator de premiação pela conservação e/ou incentivo pela adoção de práticas sustentáveis para a conservação dos remanescentes florestais. Mesmo os casos em que não há adicionalidade, agricultores da FOM que conservam um remanescente sem nunca o ter degradado poderiam ser beneficiados em algum escopo de programa de PSA, sem mencionar a manutenção dos processos ecológicos. Isso evitaria, por consequência, que apenas aqueles que geraram degradação da biodiversidade recebam o incentivo via PSA. Os programas "Desmatamento Evitado" e "Corredor Ecológico Chapecó", por exemplo, buscam a recuperação de áreas degradadas, contudo almejam a manutenção de remanescentes florestais como estratégia para valorar projetos sem o critério de adicionalidade.

Os programas de PSA devem ter como estratégia, primeiramente, o largo alcance de aplicação e valoração de seus indicadores: permanência, vazamento, adicionalidade, transação e oportunidade. $\mathrm{O}$ indicador de vazamento, em que externalidades negativas ocorrem fora da abrangência dos PSA, talvez, seja um dos indicadores que deva ser mais bem valorizado, pois serviria como parâmetro de comparação entre regiões com e sem PSA. Sugerimos que novos PSA devam planejar a permanência do programa, valorizem os envolvidos que não geram externalidades negativas (sem adicionalidade) e que acompanhem os "vazamentos". Somando isso aos demais serviços prestados, os PSA se tornarão mais eficazes, duradouros e economicamente viáveis para a atratividade de proprietários rurais e consequentes benefícios ao ambiente. Independente da tipologia envolvida num PSA, é importante que trabalhos 
futuros descrevam quais serviços ou tipologias serão relacionados no novo programa, de modo objetivo e sistemático. Os serviços culturais devem ser explicitamente citados nos programas de PSA, pois a beleza cênica, por exemplo, é uma interpretação pessoal sobre uma área conservada e de difícil valoração. É deveras importante abordar, também, junto aos futuros programas de PSA a existência de outras ferramentas de conservação que possam contemplar valores intrínsecos, bem como os serviços culturais, que motivem os proprietários a conservarem seus remanescentes também por motivações pessoais, e não somente pelo valor monetário recebido.

A Floresta Ombrófila Mista sofre com a redução de sua área de extensão original, com aumento contínuo da pressão sobre o ecossistema como um todo. O cenário de fragmentação do ecossistema, o mosaico de pequenas propriedades característico da região Sul do Brasil e o conflito de interesses entre uso de recursos e conservação são alguns exemplos acerca da complexidade dessa fitofisionomia. Os programas de PSA atuam como mecanismos locais, ou mesmo regionais, de conservação das Florestas com Araucárias, além de servirem como alternativa aos demais mecanismos utilizados pela legislação brasileira à conservação da biodiversidade, como o Código Florestal ou a Lei da Mata Atlântica, assim como ao Sistema Nacional de Unidades de Conservação (SNUC), por meio das UC de Proteção Integral e de Uso Sustentável. Ao valorar a biodiversidade para a proteção de uma espécie, e por consequência, de um ecossistema fragilizado, é inserido um forte contexto de mercantilização da biodiversidade, o que pode causar, divergências de opiniões e críticas sobre esse delicado tema. Entretanto, num cenário de pressão constante à FOM, estratégias alternativas de conservação alia- das ao uso sustentável e manutenção dos recursos favorecem e ampliam as estratégias que visam à conservação da Araucária e da Floresta Ombrófila Mista como um todo.

\section{Agradecimentos}

O presente trabalho foi realizado com apoio da Coordenação de Aperfeiçoamento de Pessoal de Nível Superior - Brasil (CAPES) - Código de Financiamento 001 (bolsa de Doutorado de MMT). Os autores gostariam de agradecer ao CNPq pela bolsa de produtividade em pesquisa para NP (Processo 310443/2015-6), à Fundação Certi pela parceria com VM, ao Fundo MAPA pelo auxílio à pesquisa. Este trabalho foi fruto da conclusão do curso de Especialização em Gestão Ambiental da UFPR por MMT e agradece a DB pelos comentários no desenvolvimento inicial desse estudo.

\section{Referências}

Adan, N.; Atchinson, J.; Reis, M. S.; Peroni, N. Local Knowledge, Use and Management of Ethnovarieties of Araucaria angustifolia (Bert.) Ktze. in the Plateau of Santa Catarina, Brazil. Economic Botany, 70(4), 353-364, 2016. doi: 10.1007/s12231-016-9361-z

AEM - Avaliação Ecossistêmica Do Milênio. Ecosystems and Human Well-being: Synthesis. Washington DC. Island Press, 2005. Disponível em < https://www.millenniumassessment.org/documents/document.356.aspx.pdf>

Alarcon G. G.; Da-Ré, M. A.; Fukahori, S. T. I. Análise de instrumentos de mercado na gestão do corredor ecológico Chapecó, Santa Catarina, Brasil. Sustentabilidade em Debate, 4(1), 117-138, 2013. Disponível em: http://periodicos. unb.br/index.php/sust/article/view/9203 
Avila, A. L. de; Araujo, M. M.; Longhi, S. J.; Schneider, P. R.; de Carvalho, J. O. P. Estrutura Populacional e regeneração de espécies arbóreas na Floresta Nacional de São Francisco De Paula, Rio Grande Do Sul. Ciência Florestal, 26(3), 825-838, 2016. doi: 10.5902/1980509824211

Bellard, C.; Leclerc, C.; Leroy, B.; Bakkenes, M.; Veloz, S.; Thuiller, W.; Courchamp, F. Vulnerability of biodiversity hotspots to global change. Global Ecology and Biogeography, 23(12), 1376-1386, 2014. doi: 10.1111/geb.12228

Bernardo, K, T. Avaliação da efetividade de esquemas de pagamentos por serviços ambientais hídricos: proposta metodológica. São Carlos, Tese (Doutorado em Ciências da Engenharia Ambiental) - Universidade de São Paulo, 2016.

Bitencourt, A. L. V.; Krauspenhar, P. M. Possible prehistoric anthropogenic effect on Araucaria angustifolia (Bert.) O. Kuntze expansion during the late Holocene. Revista Brasileira de Paleontologia, 9(1), 109-116, 2006. Disponível em: http://sbpbrasil.org/revista/edicoes/9_1/Bitencourt.pdf

Bogoni, J. A.; Graipel, M. E.; Peroni, N. The ecological footprint of Acca sellowiana domestication maintains the residual vertebrate diversity in threatened highlands of Atlantic Forest. PLoS ONE, 13(4), 2018. doi: 10.1371/ journal.pone.0195199

Brandt, M. Uma história ambiental dos campos do Planalto de Santa Catarina. Florianópolis, Tese (Doutorado em História Cultural) - Universidade Federal de Santa Catarina, 2012. Disponível em: https://repositorio.ufsc.br/xmlui/ handle/123456789/96449

Brasil. Lei no 11.428, de 22 de dezembro de 2006. Dispõe sobre a utilização e proteção da vegetação nativa do Bioma Mata Atlântica e dá outras providências. Brasília: DOU de 09/01/2007.

Carvalho, P. Espécies Florestais Brasileiras. Recomendações Silviculturais, Potencialidades e Uso da Madeira. EMBRAPA, Brasília, DF, Brasil. p. 639, 1994. Disponível em http://www.cnpf.embrapa.br/pesquisa/efb/temp/index_especies.htm

Chaudhary, S.; McGregor, A.; Houston, D.; Chettri, N. The evolution of ecosystem services: A time series and discourse-centered analysis. Environmental Science \& Policy, 24, 25-34, 2015. doi: 10.1016/j.envsci.2015.04.025
Colombo, A. F.; Joly, C. A. Brazilian Atlantic Forest lato sensu: the most ancient Brazilian forest, and a biodiversity hotspot, is highly threatened by climate change. Revista Brasileira de Biologia, 70(3), 697-708, 2010. doi: 10.1590/ S1519-69842010000400002

CONAMA - Conselho Nacional do Meio Ambiente. Resolução $n^{\circ} 278$, de 24 de maio de 2001. Dispõe sobre o corte e a exploração de espécies ameaçadas de extinção da flora da Mata Atlântica. Brasília: DOU de 18/07/2001.

Costanza, R. The value of ecosystem services: putting the issues in perspectives. Ecological Economics, 25, 67-72, 1998. doi: 10.1016/S0921-8009(98)00007-X

Da Silva, C. V.; Reis, M. S. Produção de pinhão na região de Caçador, SC: Aspectos da obtenção e sua importância para comunidades locais. Ciência Florestal, 19(4), 363-374, 2009. Disponível em: https://periodicos.ufsm.br/cienciaflorestal/article/view/892

Daly, H.; Farley, J. Ecological economics: principles and applications. International and Pan-American Copyright Conventions, Island Press, Washington DC, 2004.

Daily, G. Introduction: What Are Ecosystem Services? In: Daily, G. (Eds.). Nature's Services. Societal Dependence on Natural Ecosystems, Island Press, Washington DC, 1997.

Daily, G.; Soderqvist, T.; Aniyar, S.; Arrow, K.; Dasgupta, Partha.; Ehrlich, P. R.; Folle, C.; Jansson, A.; Jansson, B.; Kautsky, N.; Levin, S.; Lubchenco, J.; Maler, K.; Simpson, D.; Starret, D.; Tilman, D.; Walker, B. The value of nature and the nature of value. Science, 289(5478), 395-396, 2000. doi: 10.1126/science.289.5478.395

De Groot, R. S.; Wilson, M. A.; Boumans, R. M. J. A typology for the classification, description and valuation of ecosystem functions, goods and services. Ecological Economics, 41(3), 393-408, 2002. doi: 10.1016/S09218009(02)00089-7

Duarte, L. S.; Dillenburg, L. R.; Rosa, L. M. G. Assessing the role of light availability in the regeneration of Araucaria angustifolia (Araucariaceae). Australian Journal of Botany, 50, 741-751, 2002. doi: 10.1071/BT02027

Duarte, R. I.; Santos da Silva, F. A. L.; Schultz, J.; da Silva, J. Z.; Reis, M. S. Características de desenvolvimento inicial 
em teste de progênie de uma população de araucária na Flona de Três Barras-SC. Biodiversidade Brasileira, 2(2), 114-123, 2012. Disponível em: http://www.icmbio.gov.br/ revistaeletronica/index.php/BioBR/article/view/279

Engel, S.; Pagiola, S.; Wunder, S. Designing payments for environmental services in theory and practice: An overview of the issues. Ecological Economics, 65(4), 663-674, 2008. doi: 10.1016/j.ecolecon.2008.03.011

Ferro, V. G.; Lemes, P.; Melo, A. S.; Loyola, R. The reduced effectiveness of protected areas under climate change threatens Atlantic forest tiger moths. PLoS ONE, 9(9), 2014. doi:10.1371/journal.pone.0107792

Fundação Certi. Workshop Mecanismos de Investimento em externalidades ambientais positivas. Florianópolis, 2014. Não publicado.

Gasper, A.; Sevegnani, L.; Vibrans, A. Inventário florístico florestal de Santa Catarina: espécies da Floresta Ombrófila Mista. Rodriguésia, 64(2), 201-210, 2013. Disponível em: http://www.scielo.br/pdf/rod/v64n2/01.pdf

Galindo-Leal, C.; Câmara, I. G. Atlantic forest hotspots status: an overview. In: Galindo-Leal, C.; Câmara, I. G. (Eds.). The Atlantic Forest of South America: biodiversity status, threats, and outlook. pp. 3-11. Center for Applied Biodiversity Science, Island Press, Washington, D.C, 2003. Disponível em: https://escholarship.org/uc/item/56k6w49n

Guerra, M. P.; Silveira, V.; Reis, M. S.; Schneider, L. 2002. Exploração, manejo e conservação da araucária (Araucaria angustifolia). In: Simões L. L.; Lino, C. F. (Eds.). Sustentável Mata Atlântica: a exploração de seus recursos florestais. pp. 85-102. Senac, São Paulo.

Iob, G.; Vieira, E. M. Seed predation of Araucaria angustifolia (Araucariaceae) in the Brazilian Araucaria Forest: Influence of deposition site and comparative role of small and "large" mammals. Plant Ecology, 198(2), 185-196, 2008. doi: 10.1007/s11258-007-9394-6

Joly, C. A.; Metzger, J. P.; Tabarelli, M. Experiences from de Brazilian Atlantic Forest: ecological findings and conservation initiatives. New Phytologist, 204, 459-473, 2014. doi: 10.1111/nph.12989

Kanieski, M. R.; Araujo, A. C. B.; Longhi, S. J. Diversity quantification in Mixed Ombrophilous Forest by different alpha indexes. Quantificação da diversidade em Floresta Ombrófila Mista por meio de diferentes índices. Alfa, 88, 567-577, 2010. Disponível em: http://www.ipef.br/publicacoes/scientia/nr88/cap03.pdf

Lauterjung, M. B.; Bernardi, A. P.; Montagna, T.; Candido-Ribeiro, R.; Costa, N. C.; Mantovani, A.; dos Reis, M. S. Phylogeography of Brazilian Pine (Araucaria angustifolia): integrative evidence for the pre-Columbian anthropogenic dispersal. Tree Genetics \& Genome, 14(36), 2018. doi: 10.1007/s11295-018-1250-4

Lemes, P.; Loyola, R. D. Accommodating species climate-forced dispersal and uncertainties in spatial conservation planning. PLOS ONE, 8(1), 2013. doi: 10.1371/journal. pone. 0054323

Machado Mello, A. J.; Peroni, N. Cultural landscapes of the Araucaria Forests in the northern plateau of Santa Catarina, Brazil. Journal of Ethnobiology and Ethnomedicine, 11(1), 1-14, 2015. doi: 10.1186/s13002-015-0039-х

Meyer, L.; Gasper, A. L.; Sevegnani, L.; Schorn, L. A.; Vibrans, A. C.; Lingner, D. V.; Verdi, M.; dos Santos, A. S.; Dreveck, S.; Korte, A. Natural regeneration of Mixed Ombrophylous Forest in Santa Catarina. In: Vibrans, A. C., Sevagnini, L., Gasper, A. L; D. V. Liniger (Eds.). Inventário Florístico Florestal de Santa Catarina. Blumenau: Editora EdiFurb, p. 191-223, 2013. Disponível em: http://ciram. epagri.sc.gov.br/ciram_arquivos/arquivos/iff/livros/Volume\%203\%20IFFSC\%20tabelas\%20novas.pdf

Moreira, V. A. Bonificação por Produção Sustentável: estruturação e análise de indicadores de valoração para uma estratégia de Pagamento por Serviços Ambientais na Floresta com Araucárias. Florianópolis, Trabalho de Conclusão de Curso (Bacharelado em Ciências Biológicas) - UFSC, 2014.

Myers, N.; Mittermeier, R. A.; Mittermeier, C. G.; Kent, J. Biodiversity hotspots for conservation priorities. Nature, 403, 853-858, 2000. doi: 10.1038/35002501

Oliveira, E. B. Projeto Estradas com Araucárias. Livro Serviços Ambientais em Sistemas Agrícolas e Florestais do Bioma da Mata Atlântica. Emprapa, Brasília, Distrito Federal, Brasil, 2015.

Pagiola, S.; Ramírez, E.; Gobbi, J.; Haan, de C.; Ibrahim, 
M.; Murgueitio, E.; Ruíz, R. P. Paying for the environmental services of silvopastoral practices in Nicaragua. Ecological Economics, 64(2), 374-385, 2007. doi: 10.1016/j.ecolecon.2007.04.014

Peroni, N.; Albuquerque, U. P.; Assis, A. L.; Neto, E. M. F. L. The domestication of landscapes and cultural keystone species in a context of community biodiversity management in Brazil. In: Boef, S. W.; Subedi, Abishkar, Peroni, N.; Thijssen, M.; O'Keeffe, E. (Eds.). Community Biodiversity Management: promoting resilience and conservation of plant genetic resources. Routledge Press, p. 145-150, 2013. Disponível em: https://www.bioversityinternational.org/ fileadmin/user_upload/online_library/publications/pdfs/ Community_biodiversity_management/3.4.landscapes_domestication_Brazil.pdf

Plumb, S. T.; Nielsen, E. A.; Kim, Y. S. Challenges of opportunity cost analysis in planning REDD+: a honduran case study of social and cultural values associated with indigenous forest uses. Forests, 3(4), 244-264, 2012. doi: $10.3390 / \mathrm{f} 3020244$

Prado, R. B. Serviços ecossistêmicos e ambientais na agropecuária. In: Palhares, J. C. P.; Gebler, L. (Orgs.). Gestão Agropecuária. Brasília, DF, EMBRAPA, 2014, p. 413-450.

Rebollar, P. B. M.; Pasqual, J. C.; Carmo, V. B.; Loch, C. Definição de custos de oportunidade por meio da integração de dados em um Sistema de Informação Geográfica. In: Anais XVI Simposio Brasileiro de Sensoriamento Remoto - SBSR. Foz do Iguaçu, 13 a 18 de abr., 2013. Disponível em: http://www.dsr.inpe.br/sbsr2013/files/p0203.pdf

Reis, M.; Ladio, A.; Peroni, N. Landscapes with Araucaria in South America: Evidence for a cultural dimension. Ecology and Society, 19(2), 1-14, 2014. doi: 10.5751/ES06163-190243

Ribeiro, M. C.; Metzger, J. P.; Martensen, A. C.; Ponzoni, F. J.; Hirota, M. M. The Brazilian Atlantic Forest: How much is left, and how is the remaining forest distributed? Implications for conservation. Biological Conservation, 142(6), 1141-1153, 2009. doi: 10.1016/j.biocon.2009.02.021

Ribeiro, M. C.; Martensen A. C.; Metzger, J. P.; Tabarelli, M.; Scarano, F.; Fortin, M-J. The Brazilian Atlantic Forest: a shrinking biodiversity hotspot. In: Zachos, F. E.; Habel, J. C.
(Eds.). Biodiversity Hotspots - Distribution and Protection of Conservation Priority Areas. Springer, p. 405-434, 2011. doi: 10.1007/978-3-642-20992-5

Ribeiro, T. M.; Ivanauskas, N. M.; Martins, S. V.; Polisel, R. T.; dos Santos, R. L. R. Fitossociologia de uma Floresta Secundária com Araucaria angustifolia (Bertol.) O. Kuntze na Estação Ecológica de Bananal, Bananal-SP. Floresta e Ambiente, 20(2), 159-172, 2013. Disponível em: http:// www.scielo.br/pdf/floram/v20n2/a03v20n2.pdf

Roderjan, C. V.; Galvão, F.; Kuniyoshi, Y. S.; Hatschbach, G. G. As unidades fitogeográficas do Estado do Paraná. Ciência \& Ambiente, 24, 76-92, 2002.

Santos, F. L.; Silvano, R. A. M. Aplicabilidade, potenciais e desafios dos Pagamentos por Serviços Ambientais para conservação da água no sul do Brasil. Desenvolvimento e Meio Ambiente, 38, 481-498, 2016. doi: 10.5380/dma. v38i0.43640

Scarano, F. R.; Ceotto, P. Brazilian Atlantic forest: impact, vulnerability, and adaptation to climate change. Biodiversity and Conservation, 24(9), 2319-2331, 2015. doi: 10.1007/ s10531-015-0972-y

SCRural - Santa Catarina Rural. Cooperação para o Desenvolvimento Rural. Governo de Santa Catarina. 2014. Disponível em: $<$ http://www.scrural.sc.gov.br/?cat=480/>. Acesso em: mai. 2017.

Souza, A. F.; Matos, D. U.; Forgiarini, C.; Martinez, J. Seed crop size variation in the dominant South American conifer Araucaria angustifolia. Acta Oecologica, 36, 126-134, 2010. doi: 10.1016/j.actao.2009.11.001

Reis, M.; Ladio, A.; Peroni, N. Landscapes with Araucaria in South America: Evidence for a cultural dimension. Ecology and Society, 19(2), 2014. doi: 10.5751/ES-06163-190243

Seehusen, S. E.; Cunha, A. A.; Oliveira, A. F. Jr. Iniciativas de PSA de Proteção da Biodiversidade na Mata Atlântica. In: Guedes, F.B.; Seehusen, S. E. (Orgs.). Pagamentos por Serviços Ambientais na Mata Atlântica: lições aprendidas e desafios. Brasília: MMA, 2011. Disponível em: $<$ http:// www. mma.gov.br/estruturas/202/_arquivos/psa_na_mata atlantica_licoes_aprendidas_e_desafios_202.pdf, p. 17>. Acesso em: abr. 2017. 
SPVS - Sociedade de Pesquisa em Vida Selvagem e Educação Ambiental. Mecanismos para o combate às mudanças climáticas e conservação de ambientes naturais. 2013. Disponível em: <http://spvs.org.br/redd/pt-cap3.pdf>. Acesso em: abr. 2017.

Vibrans, A. C.; Sevegnani, A.; Uhlmann, L. A.; Schorn, L. A.; Sobral, M. G.; Gasper, A. L.; Lingner, D. V.; Brogni, E.; Klemz, G.; Godoy, M. B.; Verdi, M. Structure of mixed ombrophyllous forests with Araucaria angustifolia (Araucariaceae) under external stress in southern Brazil. Revista de Biologia Tropical, 59, 1371-1387, 2011. Disponível em: http://ref.scielo.org/gxv84x

Vibrans, A. C.; McRoberts, R. E.; Moser, P.; Nicoletti, A. L. Using satellite image-based maps and ground inventory data to estimate the area of the remaining Atlantic forest in the Brazilian state of Santa Catarina. Remote Sensing of Environment, 130, 87-95, 2013. doi:10.1016/j.rse.2012.10.023

Vieira da Silva, C. V.; M. S. Reis. Produção de pinhão na região de caçador: aspectos da obtenção e sua importância para comunidades locais. Ciência Florestal, 19(4), 363-374, 2009. doi: $10.5902 / 19805098892$

White, D.; Minang, P. (Eds.). Estimating the Opportunity Costs of REDD+. A training manual. Washington: The World Bank, 2011. Disponível em: <http://documents. worldbank.org/curated/en/747421468337285819/pdf/632690WP0Oppor00Box0361511B0PUBLIC0.pdf>. Acesso em: nov. 2017.

Wrege, M. S.; Sousa, V. A.; Fritzsons, E.; Soares, M. T. S.; Aguiar, A. V. Predicting current and future geographical distribution of Araucaria in Brazil for fundamental niche modeling. Environment and Ecology Research, 4(5), 269279, 2016. doi: 10.13189/eer.2016.040506

Wunder, S. Payments for environmental services: Some nuts and bolts. CIFOR Occasional Paper, 2005. Disponível em: https://www.cifor.org/publications/pdf_files/OccPapers/ OP-42.pdf

Wunder, S. Revisiting the concept of payments for environmental services. Ecological Economics, 117, 234-243, 2015. doi: 10.1016/j.ecolecon.2014.08.016

Wunder, S.; Börner, J.; Tito, M. R.; Pereira, L. Pagamentos por serviços ambientais: perspectivas para a Amazônia
Legal. Estudos Series N. 10. Ministério do Meio Ambiente (MMA), Brasília, p.136, 2009. Disponível em: http://www. mma.gov.br/estruturas/168/_publicacao/168_publicacao17062009123349.pdf

Young, C. E. F.; Mac-Knight, V.; Meireles, A. L. Desmatamento e custo de oportunidade da terra: o caso do Mato Grosso. In: Anais VII Encontro Nacional da Sociedade Brasileira de Economia Ecológica. Fortaleza, 28 a 30 de nov., 2007. Disponível em: <http://www.ecoeco.org.br/>. Acesso em: nov. 2017.

Young, C. E. F.; De Bakker, L. B. Payments for ecosystem services from watershed protection: A methodological assessment of the Oasis Project in Brazil. Natureza e Conservação, 12(1), 71-78, 2014. doi: 10.4322/natcon.2014.013

Zechini, A. A.; Lauterjung, M. B.; Candido-Ribeiro, R.; Montagna, T.; Bernardi, A. P.; Hoeltgebaum, M. P.; Mantovani, A.; Reis, M. S. Genetic Conservation of Brazilian Pine (Araucaria angustifolia) Through Traditional Land Use. Economic Botany, 20(10), 1-14, 2018. doi: org/10.1007/ s12231-018-9414-6 